\title{
Serological Assessment of COVID-19 Patients in Brazil: Levels, Avidity, and Subclasses of IgG Against RBD
}

\author{
Andrew Moura \\ Instituto Adolfo Lutz \\ Hernan H. da Costa \\ Instituto Adolfo Lutz \\ Victor Correa \\ Instituto Adolfo Lutz \\ Ana K. Lima \\ Instituto de Infectologia Emilio Ribas \\ José A. Lindoso \\ Universidade de São Paulo \\ Elizabeth De Gaspari \\ Instituto Adolfo Lutz \\ Marisa Hong \\ Instituto Adolfo Lutz \\ Jair Cunha-Junior \\ Universidade Federal de Uberlândia \\ Carlos Prudencio ( $\nabla$ carlos.prudencio@ial.sp.gov.br) \\ Center of Immunology, Institute Adolfo Lutz, São Paulo, SP, Brazil
}

\section{Research Article}

Keywords: SARS-COV-2, COVID-19, Serological assay, ELISA, RBD

Posted Date: January 8th, 2021

DOl: https://doi.org/10.21203/rs.3.rs-131195/v1

License: (c) (1) This work is licensed under a Creative Commons Attribution 4.0 International License.

Read Full License 


\section{Abstract}

SARS-CoV-2 is considered a global emergency, resulting in an exacerbated crisis in the health public in the world. Although there are advances in vaccine development, it is still not available for many countries. On the other hand, an immunological response that mediates protective immunity or indicates that predict disease outcome in SARS-CoV-2 infection remains undefined. This work aimed to assess the antibody levels, avidity, and subclasses of IgG to RBD protein, in symptomatic patients with severe and mild forms of COVID-19 in Brazil using an adapted in-house RBD-IgG ELISA. The RDB IgG-ELISA showed $100 \%$ of specificity and $94.3 \%$ of sensibility on detecting antibodies in the sera of hospitalized patients. Patients who presented severe COVID-19 had higher anti-RBD IgG levels compared to patients with mild disease. Additionally, most patients analyzed displayed low antibody avidity, with $64.4 \%$ of the samples of patients who recovered from the disease and $84.6 \%$ of those who died in this avidity range. Our data also reveals an increase of IgG1 and IgG3 levels since the 8th day after symptoms onset, while IgG4 levels maintained less detectable during the study period. Surprisingly, patients who died during 8-14 and 15-21 days also showed higher anti-RBD IgG4 levels in comparison with the recovered $(P<0.05)$, suggesting that some life-threatening patients can elicit IgG4 to RBD antibody response in the first weeks of symptoms onset. Our findings constitute the effort to clarify IgG antibodies' kinetics, avidity, and subclasses against SARS-CoV-2 RDB in symptomatic patients with COVID-19 in Brazil, highlighting the importance of IgG antibody avidity in association with IgG4 detection as tool laboratory in the follow-up of hospitalized patients with more significant potential for life-threatening.

\section{Introduction}

COVID-19 (Coronavirus disease 2019), the most recent pandemic caused by severe acute respiratory syndrome-related coronavirus 2 (SARS-CoV-2), resulting in an exacerbated crisis in the health public, declared as a global emergency by World Health Organization (WHO) ${ }^{1}$ in March 2020. SARS-CoV-2 was first reported in Wuhan, China, in December 2019, resulting in a large number of individuals presenting symptoms such as fever, dry cough, dyspnea, body pain, loss of taste and smell, and sometimes atypical pneumonia that might be fatal in a small percentage of cases (around 5\%) ${ }^{2,3,4}$. Brazil became the epicenter of COVID-19 ${ }^{5}$ in June of 2020 , averaging about 1.000 deaths per day ${ }^{6}$. After a gradual reduction in the number of deaths from COVID-197, nowadays Brazil has experienced a rise in the number of cases and deaths associated with SARS-CoV-2 infection. This scenario may impose new challenges to health services shortly, including the requirement for novel rapid diagnostic tools to interrupt the COVID19 epidemiological chain ${ }^{8}$.

SARS-CoV-2 is a novel human-infecting Betacoronavirus that is enveloped, non-segmented, positivesense single-stranded RNA of around 65-125 nm diameter with crown-like spikes proteins on its outer surface ${ }^{9}$. The new coronavirus genome encodes nonstructural proteins from two open reading frames 1a and $1 \mathrm{~b}$ (Open reading frame 1 - ORF1), and structural proteins, such as spike (S), small envelope (E), membrane $(\mathrm{M})$, and nucleocapsid $(\mathrm{N})$ glycoprotein ${ }^{10}$. The $\mathrm{S}$ glycoprotein is a transmembrane protein 
with a molecular weight of $150 \mathrm{KDa}$ that binds to the angiotensin-converting enzyme 2 (ACE2) or CD147 receptor expressed on the host cells surface through receptor binding protein (RBD) presented in the S1 subunit of $S$ protein ${ }^{11,12}$.

Although the gold-standard diagnosis test is based on detecting viral nucleic acid by real-time reverse transcription-polymerase chain reaction (RT-PCR) assay, serological tests as anti-COVID-19 IgG/lgM rapid tests have been applied mainly as an essential diagnostic tool to evaluate the seroprevalence of SARSCoV2. Nevertheless, their accuracy is inferior to ${ }^{13,14,15}$. Therefore, the development of new serological tests is an urgent need because RT-PCR, used for disease diagnosis with high sensitivity and specificity, can only detect virus from pharyngeal and saliva specimens samples from the first week up to 10 days after symptoms onset ${ }^{16,17}$, and asymptomatic subjects can be missed from this screening. Also, manipulations of potentially infectious materials, which may cause splashes, droplets, or aerosols, are highly recommended to be done in a biosafety level - 2 (BSL-2) structure at least, besides RT-PCR is a laborious and expensive technique 18,19,20,21. $^{\text {1 }}$

Studies reveal that the higher infectivity capacity of SARS-CoV-2 compared to other coronavirus is due to genomic mutations on the RBD domain from the $S$ protein that plays an essential role in virus attachment and invasion to the host cell ${ }^{22}$. On the other hand, because the RBD domain is highly immunogenic and induces $\lg G$ antibody response in acutely infected patients, it is considered a potential target for serological assays ${ }^{23-29}$ and vaccine development ${ }^{10,30,31}$. Although there are advances in vaccine development ${ }^{32,33}$, it is still not available for many countries. On the other hand, the immunological response in SARS-CoV-2 infection remains undefined, with several data still emerging in the scientific literature concerning this new coronavirus pandemic ${ }^{34,35,36}$

While virus nucleic acid is detected between 3-10 days after infection, antibody production can only be detected at least 7 days after symptoms onset, similar to SARS-CoV-1 infection ${ }^{37}$. A high level of antibodies is observed in patients with severe disease ${ }^{38,39}$. However, data regarding their ability to neutralize infection is missing, and the presence of $\lg G$ subclasses $\lg G 1, \lg G 2$, IgG3, and $\lg G 4)$. In contrast, some recovered, or asymptomatic patients produce low or absence of IgG levels against nucleoprotein and spike protein from SARS-CoV- $2^{40}$.

Antibodies IgG1 and IgG3 are related to complement fixation, antibody-dependent cellular toxicity, and viral neutralization ${ }^{41}$, and a recent study has demonstrated that IgG1 and IgG3 specific to RBD occurs primarily in COVID-19 patients during acute infection. At the same time, IgG2 and IgG4 are less detectable in patients sera ${ }^{29}$, suggesting that IgG subclasses as an important marker to distinguish disease timepoint and, perhaps, disease severity. Other factors can also contribute to the understanding of humoral responses to SARS-CoV-2 and the role of neutralizing antibodies in the convalescent plasma of donors, such as an association between antibody neutralizing titers and antibody avidity ${ }^{42}$. Antibodies produced by patients that recovered from coronavirus disease 2019 (COVID-19) have been used as a "molecular framework" to design antibody-based therapies ${ }^{43}$. Thus, the study aimed to evaluate the kinetic, level, and 
avidity of antibodies IgG RBD-specific and the relationship of specific IgG subclasses to the severity of the disease.

\section{Results}

\section{Demographic data, clinical aspects, and comorbidities.}

Forty-seven symptomatic patients tested positive for SARS-CoV2 infection admitted at the Institute of Infectology Emilio Ribas (IIER; São Paulo, Brazil) between March and June 2020 enrolled in this study. The clinical characteristics of these patients are shown in Table 1. The median age was 59 years (IQR, 33-82 years); $61.7 \%$ were males, and $38.3 \%$, females. The most common symptoms at illness onset were fever (39 [82.98\%]), dry cough (39 [82.98\%]), dyspnea (34 [72.34\%]) and myalgia (20 [42.55\%]). Obesity (25 [53.19\%]), hypertension (23 [48.94\%]) and diabetes (16 [30.04\%]) were the most common comorbidities. Inpatients were seen at the Intensive Care Unit, and some of them, due to severity, required intubation (37-78.72\%). The pharmacological treatment varied according to clinical features and coexisting disorders of each patient: 45 patients (95.74\%) received antibiotics, 24 (51.06\%) antiviral, 16 (30.04\%) corticoids, $13(27.67 \%)$ anticoagulants, and 9 (19.15\%) chloroquine (Table 1). Most of the patients recovered from COVID-19 (35/47 [74.5\%]), in which 25 (71.43\%) developed the severe form of illness, while $10(28.57 \%)$ developed the mild form of the disease. Obesity, hypertension, and diabetes were predominant comorbidities observed in both symptomatic patients who recover or pass away.

\section{IgG RBD-specific antibodies detected by in house ELISA}

An adapted in house ELISA using RBD as coat antigen was performed to detect specific IgG in serum samples from SARS-CoV-2 symptomatic patients. A total of 294 serum samples were analyzed, being 176 serum samples collected on different days from 47 SARS-CoV-2 positive patients admitted to the Institute of Infectology Emilio Ribas (IIER) and 118 negative serum samples from Institute Adolfo Lutz (IAL) routine - (collected before 2019). Among SARS-CoV2 positive patients, IgG RBD-specific antibodies were detected in $166(94.32 \%)$ samples, while none SARS-CoV2 negative controls $(n=118)$ showed reactivity (Fig. 1a). The sample from patients of different time points (intervals of 7 days) post-onset symptoms were analyzed (Fig. 1a) to assess the immunoreactivity throughout the time. Results had shown only $50 \%$ of samples ( 5 out of 10 ) from SARS-CoV- 2 infected patients presented IgG RBD-specific positivity within the first-week post the onset of the symptoms, with no significant difference compared to negative controls.

Otherwise, from the second week on after onset symptoms, the presence of IgG RDB-specific antibodies was observed among $88.6 \%$ of patient's samples, reaching $100 \%$ of positivity in the next time-point, highlighting that the sensitivity of RBD-based ELISA improves according to the timing after the onset of clinical symptoms (Fig. 1b-h). The general immunoreactivity of RDB ELISA (including first and second week) resulted in $100 \%$ of specificity and $94.3 \%$ of sensibility in the population analysed (Fig. 1). 


\section{IgG SARS-CoV-2 RBD-specific antibodies according to the outcome (recovered or died)}

We analyzed the IgG antibody response against SARS-Cov-2-RBD using samples from patients collected at least 15 days after symptoms onset, grouped according to the patient's outcome: recovered (group 1, $\mathrm{n}=35$ ) and died (group 2, $\mathrm{n}=12$ ). The significant comorbidities observed in patients who died were obesity (8/12 [66.67\%]), followed by hypertension (6/12 [50\%]) and diabetes (5/12 [41.67\%]) (Fig. 2a). The group of patients who died displayed a slightly lower anti-RBD IgG level than the recovered one, although no differences were observed in both groups (Fig. 2b). Interestingly, patients with severe COVID19 had higher anti-RBD IgG levels than patients with mild disease $(P<0.05$, Fig. $2 C)$.

\section{IgG antibody avidity to SARS-CoV-2 RBD}

Samples collected from 15 days after onset of symptoms from patients with COVID-19 who symptoms were evaluated for binding strength of IgG antibodies to RBD protein. The majority of patients analyzed displayed low antibody avidity, with $64.4 \%$ of the samples of patients who recovered from the disease and $84.6 \%$ of those who died in this avidity range (Fig. 3a). Intermediate antibody avidity was observed in $33.3 \%$ and $15.4 \%$ in the recovered or died group, respectively. Remarkably, one sample from a patient that recovered displays high avidity to SARS-CoV2 RBD, despite a relatively short period (weeks) posts the onset of the symptoms. When the samples were analyzed in parallel, in each avidity range (low, intermediate, high), no differences were detected between patients who recovered or died from covid-19 (Fig. 3a). Similarly, when samples from patients who recovered or died were analyzed each week (7-day intervals) comparatively, no statistically significant difference was observed (Fig. 3b).

Antibody subclasses response in symptomatic patients with COVID-19

IgG subclasses maybe become relevant in clinical conditions COVID-19, considering that IgG subclasses to SARS-CoV-2 are a key to a better clinical condition, with IgG1 and IgG3 be more abundant in patients that are in the mild case and do not die. Our data reveal an increase of IgG1 and IgG3 levels since the 8th day after symptoms onset (Fig. 4a), while IgG4 levels maintained less detectable during the study period. Anti-RBD IgG1 positivity oscillated from $66.6-100 \%$, reaching the highest values in the third and fourth week of analysis. The positivity of anti-RBD IgG3 ranged from $66.6-90.9 \%$, whereas IgG4 presented positivity from 66.6 to $46.1 \%$, with higher values for IgG3 and IgG4 positivity observed in the third and fourth weeks, respectively.

When patients who died and who recovered were analyzed in parallel, it was possible to identify a higher anti-RBD IgG1 response in patients who died compared to those who recovered during 8-14 days after symptoms onset $(P<0.05)$, as illustrates in the Fig. $4 \mathrm{~b}$. No statistical differences were observed in IgG3 levels from recovered and dead patients (Fig. 4c). Surprisingly, patients who died during 8-14 and 15-21 days also showed higher anti-RBD IgG4 levels in comparison with the recovered $(P<0.05)$ (Fig. $4 d)$, 
suggesting that some life-threatening patients can elicit IgG4 to RBD response in the first weeks of symptoms onset.

\section{Discussion}

SARS-CoV-2 exhibits a high infectivity capacity; the new disease reached the mark of 65.8 million cases worldwide, being observed more than 1,5 million deaths ${ }^{7}$. Brazil is entering through the second wave of COVID-19, and some restrictions are being imposed. However, the number of cases is still climbing. In the absence of vaccines and a useful serological assay to trial even asymptomatic people, SARS-CoV-2 infection remains a significant problem affecting Brazil. Although qPCR to detect the viral genome is a gold standard diagnostic assay, additional screening methods that detect the presence of SARS-Cov-2 infection, including serological tests, can be highly advantageous to ensure timely diagnosis in a pandemic ${ }^{31}$. Several studies have reported the profile of the antibody response to SARS-CoV-2, which includes the broad clinical spectrum of COVID-19. Many aspects of the humoral immune response in COVID-19 remain obscure ${ }^{44,45-47}$, particularly concerning clinical utilities of serological testing in symptomatic and hospitalized patients.

In this attempt, we evaluated the IgG response in hospitalized patients with mild and severe clinical manifestation, showing RBD-specific IgG antibody responses begins 4 days after symptoms onset, reaching the plateau at 15 days. The average time to detect IgG antibodies against SARS-CoV-2 based on RBD IgG-ELISA was similar to what was found in other studies, regardless of the serological assay and SARS-CoV-2 antigen used $44,45-47$. Our in-house ELISA's high immunoreactivity was observed with $100 \%$ specificity and $94.3 \%$ of sensibility, suggesting that RDB IgG-ELISA could be used to assess SARS-CoV-2 infection in patients 15 day-post onsets of symptoms. Wolf at. al; 2020 observed that hospitalized COVID-19 patients showed higher levels of antibodies when compared to outpatients cases ${ }^{43}$. A previous report demonstrated that hospitalized COVID-19 patients showed higher levels of antibodies than outpatients cases ${ }^{43}$, suggesting an association between illness severity and antibody production.

Interestingly, in our study, survivors who developed the severe form of illness displayed higher anti-RBD IgG levels compared to patients with mild disease, also lighting that clinical presentation of disease may produce substantial differences in IgG responses. For this purpose, we also investigated the anti-RBD IgG levels in recovered patients compared to those who died. Although no statistical differences were observed, a slightly lower anti-RBD IgG level was observed in patients who died compared to the recovered ones. The divergence observed in our study may be due to differences in the number of serum samples analyzed and the time of blood collection in our study since a limited number of samples was obtained from critically ill patients who quickly progressed to death by COVID-19.

Besides the comorbidities observed in patients who died, obesity was more frequently observed in our study. The high percentage of obese patients who died with COVID-19 is in concordance with previous studies ${ }^{48,49}$. Obesity is a factor that directly associated chronic activation of innate immune system cells and consequent local and systemic inflammation ${ }^{50}$. B cells from obese patients express leptin induced- 
activation markers (TNF-a, TLR4, micro-RNAs) that correlate reduced B-cell functions ${ }^{49,51,52}$. Therefore, obesity and COVID-19 share common elements of the inflammatory process (and possibly also metabolic disturbances), exacerbating SARS-CoV-2 infection in the obese ${ }^{53}$, leading these individuals to severe COVID-19, even to death.

In the present study, high levels of anti-RBD IgG were detected in both groups of patients who died or survived. However, these findings are not enough to support the hypothesis that these individuals displayed either an extended-lasting protective humoral response against SARS-CoV-2 or neutralizing antibodies in convalescent plasma. Our study has some limitations in this context since it was not possible to assess the presence and neutralizing antibodies. Interestingly, a possible association between SARS-CoV-2 spike antibody avidity with neutralizing IgG titter, as a potential screening parameter for identifying optimal convalescent plasma donors was proposed ${ }^{42}$. Likewise, high-avidity antibodies toward another virus capable of blocking receptor binding were protective and promoted virus neutralization ${ }^{54}$, indicating that antibody avidity maturation could be, at least in part, associated with the production of protective neutralizing antibodies in viral infections. Although our study has limited data on temporal dynamics (< 45 days) to correlates SARS-CoV-2 antibody avidity with the illness severity, it was observed that the majority of patients who had symptoms showed low avidity. Our data is by what was reported to SARS-CoV-2 infection with low IgG antibody avidity during the 50 days after symptoms onset 42 , however, evaluating antibodies against nucleoprotein ${ }^{55}$, and to SARS-CoV-2 anti-spike and antinucleoprotein, that reached the avidity peak at 21 after days of symptoms onset, during a study period median of 45 days ${ }^{42}$.

Similarly, low antibody avidity was also observed early infection and augmented within the first month of symptom onset in SARS outbreaks ${ }^{56}$. It is noteworthy that one-third of the patients who recovered had intermediate avidity of IgG antibodies to RDB, despite a relatively short period (weeks) post the onset of the symptoms. On the other hand, approximately one-sixth of the patients who died produced intermediate avidity antibody responses, suggesting that IgG avidity may be useful for monitoring hospitalized patients with COVID-19 in association with other serological markers. As was expected, IgG antibody avidity was low during initial infection and increased with time, although no statistical differences were observed between patients who died and recovered in the time-points post the onset of the symptoms.

The IgG1 and IgG3 subclasses represent the predominant antibody responses to several viral diseases 57,41 , and recently it was also associated with the new SARS-CoV -2 infection ${ }^{29,58}$. IgG1 and IgG3 responses are related to immune functions such as viral neutralization, opsonization, and complement activation in viral respiratory infection ${ }^{41}$. Thus, to further analyze the antibody response, we also analyzed IgG to SARS-CoV-2 RDB in sera from patients with COVID-19. As it was expected, a robust antibody response of IgG1 and IgG3 specific to SARS-CoV-2 RDB occurred predominantly in comparison with minor IgG4 responses. Likewise, Suthar et al.; 2020 demonstrated that COVID-19 patients analysed in USA produced RBD-specific IgG1 and IgG3 early during acute infection, with no detectable IgG2 or 
$\lg 4^{29}$. Similar antibody responses were also reported by Mazzini et al.; 2020 in Italy, with a strong reactivity for IgG1 and IgG3 in sera from positive patients for SARS-CoV-2 infection ${ }^{58}$.

The comparative analysis of IgG subclasses in serum samples from COVID-19 patients who died revealed a higher level of RDB-specific IgG1 when compared to those who survived during 0-8 days after symptoms onset. However, this difference was not maintained in more advanced times of the onset of the symptoms. Although the production of RDB-specific lgG1 is consistent with activation of type $1 \mathrm{~T}$ helper lymphocytes (Th1) ${ }^{59}$, this focal difference in the first week cannot be explained simply by balancing different subpopulations of T helper cells but maybe involve other factors, including sample bias, differences in individual immune responses, and/or early viral load. Also, no statistical differences were observed in IgG3 levels between patients who died and recovered. Surprisingly, we also noticed higher levels of RBD-specific IgG4 in sera from patients who died when compared to survivors in the second and third weeks of analysis. In our analysis, $55 \%$ (10 out of 18) serum samples of patients who progressed to death showed early positivity to RDB-specific IgG4 antibodies, whereas patients who recovered from COVID-19 were IgG4 negative to SARS-CoV-2 RDB in the same window of time. The IgG4 biosynthesis is known to be induced under conditions of increased IL-10 cytokine ${ }^{60}$ having as a primary source several immune cells, including Th2 cells, regulatory T cells (Treg), or even regulatory B cells (Breg). Patients with severe COVID-19 display sustained inflammation and continued production of various anti- and pro-inflammatory cytokines (cytokine storm syndrome) ${ }^{36}$, including the IL-10 production that may be associated with induction of IgG4 antibodies in severe COVID-19. Although substantial knowledge about the antibody response has already been generated nowadays for COVID-19, further studies are necessary to understand the role of IgG4 antibodies in COVID-19 pathophysiology.

In conclusion, the present study constitutes the effort to clarify the kinetics of IgG antibodies, avidity, and subclasses against SARS-CoV-2 RDB in symptomatic patients with COVID-19 in Brazil, highlighting the importance of IgG antibody avidity in association with IgG4 detection as a laboratory tool in the follow-up of hospitalized patients with more significant potential for life-threatening conditions in the population analyzed.

\section{Methods}

\section{Study design}

Forty-seven symptomatic patients tested positive for SARS-CoV2 infection by RT-PCR were admitted at Institute of Infectology Emilio Ribas (IIER) São Paulo, Brazil, between March and June 2020 were enrolled in this study. All patients who presented typical symptoms of illness such as fever, dry cough, dyspnea, myalgia, etc., were classified as mild or severe, according to IIER protocols previously established for COVID-19. Blood samples were taken at different time points until either the patient was discharged or died. A total of 294 serum samples were used from patients (136 collected from patients who were discharged and 40 from patients who died) and 118 serum samples SARS-CoV2 negative, collected 
before September 2019 and selected from Institute Adolfo Lutz (IAL) routine, were analyzed (Fig. 5). SARS-CoV2 negative serum samples have a documented history of other viral infections (HIV-1, HIV-2, Hepatitis B, Hepatitis C, Dengue, Chikungunya, Yellow fever) non-related with any coronavirus and bacterial infections (Treponema pallidum, Mycoplasmas pneumoniae).

\section{Ethical Approvements}

The Committee for Ethics approved clinical research from both Institute of Infectology Emilio Ribas and Institute Adolfo Lutz, CAAE number: 35589320.6.3001.0075. All methods were performed in accordance with the relevant guidelines and regulations. Human samples used in this study correspond to discarded peripheral blood, collected previously for monitoring hematological characteristics of patients with suspected SARS-CoV2 infection. Demographic data, Clinical and hematological conditions at first attendance were obtained for all patient included in the study.

\section{SARS-CoV-2 RBD recombinant antigen}

The receptor-binding domain (RBD) antigen, derived from SARS-CoV2 Spike protein, was kindly donated by Dr. Florian Krammer ${ }^{23,24}$, from Icahn School of Medicine, Mount Sinai, Nova York, NY, EUA.

\section{Indirect ELISA for detection of SARS-CoV-2 RBD-specific IgG antibodies (ELISA-RBD)}

IgG RBD-specific antibodies were detected by Enzyme-Linked Immunosorbent Assay (ELISA) using an adapted protocol previously described by Stadlbauer et al. ${ }^{24}$. Briefly, high-binding 96-well plates (Nunc MaxiSorp $^{\mathrm{TM}}$ flat-bottom) were coated with $50 \mu \mathrm{l}$ per well of $2.5 \mu \mathrm{g}$ per $\mathrm{ml}$ of RBD protein diluted in PBS $1 \mathrm{x}$ at $4^{\circ} \mathrm{C}$ overnight. The next morning, plates were washed four times with PBS $1 \mathrm{x}$ supplement with $0.01 \%$ Tween 20 (PBST). All wash steps were performed using an ELISA plate washer (Washwell plate, Robonik, Thane, India). After, $200 \mu \mathrm{l}$ per well of $5 \%$ skim milk powder diluted in PBST was added to the plates and incubated for $2 \mathrm{~h}$ at room temperature as a blocking solution. After blocking, plates were washed four times with PBST and incubated with $200 \mu \mathrm{l}$ per well of each sera sample, in duplicate, diluted 1:200 in PBST containing $1 \%$ of skin milk for $1 \mathrm{~h}$ at room temperature. Next, plates were washed four times with PBST and $50 \mu$ of a 1:15000 dilution of goat anti-human IgG (whole molecule) - Horseradish Peroxidase (HRP) antibody (Sigma-Aldrich) diluted in PBST containing 1\% skim milk was added to wells, and the plates incubated for $1 \mathrm{~h}$ at room temperature. Plates were rewashed with PBST and incubated for 10 minutes with $100 \mu$ l of One Step-TMB (3,3',5,5'- tetramethylbenzidine) (Scienco, Santa Catarina, Brazil). The reaction was stopped by the addition of $50 \mu$ l per well of $1 \mathrm{~N}$ sulfuric acids. The optical density at $450 \mathrm{~nm}$ (OD450) was measured using a Multiskan MS plate reader (Labsystems). The cut-off value was established based on the maximum sensitivity and specificity using a two-graph receiver operating characteristic (TG-ROC) analysis as previously described ${ }^{61}$. Antibody titers were expressed as ELISA index $(E I)$, according to the following formula: $E I=O D$ sample/cut-off. Samples with El values $>1.0$ were considered positive.

\section{Evaluation of IgG antibody avidity to SARS-CoV-2 RDB}


Serum samples were submitted to IgG avidity ELISA using potassium thiocyanate (KSCN) as a chaotropic chemical reagent as previously described ${ }^{62}$ to assess the interaction between them IgG antibodies and RBD. Following the above described ELISA-RBD, an extra step was performed after incubation with serum. Briefly, after serum incubation, plates were washed four times with PBST, and wells were treated incubated in the presence or absence of KSCN 1.5M (200 $\mu \mathrm{l} /$ well) for 20 minutes at room temperature. After, plates were washed four times and. Avidity index (Al\%) was expressed as follows: $\mathrm{Al} \%=(\mathrm{OD}$ mean value from $\mathrm{KSCN}$ treated sample divided by the OD mean value from the nontreated) multiplied by 100 . Al values above $50 \%$ were considered high antibody avidity; between $31-49 \%$, intermediate avidity, and below $30 \%$, low avidity ${ }^{63}$.

\section{Measurement of IgG specific to SARS-CoV-2 RDB}

ELISA was used to detect IgG1, IgG3, IgG4 using a protocol previously described ${ }^{64}$. Briefly, 96-well plates (Nunc MaxiSorp ${ }^{\mathrm{TM}}$ flat-bottom) were coated with $50 \mu \mathrm{l}$ per well of $2.5 \mu \mathrm{g}$ per $\mathrm{ml}$ of RBD protein diluted in PBS $1 x$ at $4^{\circ} \mathrm{C}$ overnight. Plates were then washed four times with PBS $1 \mathrm{x}$ with $0.01 \%$ Tween 20 (PBST) using a Washwell Plate (Robonik). Samples were blocked with $100 \mu$ l per well of $1 \%$ Bovine Serum Albumin (BSA) diluted in PBST for one $\mathrm{h}$ at $37^{\circ} \mathrm{C}$. After blocking, plates were washed four times with PBST and incubated with serum diluted 1:50 in PBST-0,1\% BSA, in duplicate, for IgG1 detection. For IgG3 and IgG4 subclasses, serum was diluted 1:5 in the same solution, and the assay was also performed in duplicate. Thus, samples were incubated for $2 \mathrm{~h}$ at $37^{\circ} \mathrm{C}$, washed four times, and incubated with the respective biotinylated secondary antibodies (Sigma): goat anti-human IgG1 (1:1000), anti-human IgG3 (1:1000), or anti-human IgG4 (1:1000) diluted in PBST $-0.1 \%$ BSA for $1 \mathrm{~h}$ at $37 \circ \mathrm{C}$. Plates were rewashed four times and incubated with $50 \mu$ of 1:500 streptavidin-peroxidase (Sigma/Merck) diluted in PBST $0.1 \% \mathrm{BSA}$ (Sigma) for $30 \mathrm{~min}$ at $37^{\circ} \mathrm{C}$. After the final washing step (four times), samples were revealed with ABTS (Sigma/Merck). The optical density at $405 \mathrm{~nm}$ (OD405) was measured using a Multiskan MS plate reader (Labsystems). Cut-off of reaction was calculated using optical density values of negative

pools plus three standard deviations as described ${ }^{59}$. Antibody titers were expressed as ELISA index (EI), and values $>1.0$ were considered positive.

\section{Statistical Analyses}

The data were evaluated for normal distribution by D’Agostino \& Pearson, Shapiro-Wilk, KolmogorovSmirnov normality tests. Statistically, differences among antibody IgG levels, antibody avidity, and IgG subclasses to SARS-CoV-2 RB were determined by Kruskal-Wallis and Dunn's multiple comparisons test or Mann-Whitney test when appropriate. $P$ values $<0.05$ were considered statistically significant ${ }^{65}$. Statistical analyses and graphics were performed using the GraphPad Prism v. 8.0 (GraphPad Software, San Diego, USA).

\section{Declarations}

Acknowledgements 
We thank Dr. Florian Krammer from Icahn School of Medicine, Mount Sinai, Nova York, NY, EUA for donating RBD recombinant protein. We also thanks Dr. Marcela de Azevedo from Adolfo Lutz Institute for revising the manuscript. This work was supported by grants from National Council for Scientific and Technological Development (CNPq Grant ID 440812/2016-0), Ministry of Health, Coordination for the Improvement of Higher Education Personnel (CAPES Grant ID 88881.130804/2016-01) and the São Paulo Research Foundation (FAPESP Grant ID 17/50333-7) to (CRP) and (FAPESP Grant ID 18/04202-0) to (EDG).

\section{Authors contributions}

A.D.M., E.D.G., M.A.H.; J.A.L.L., C.R.P., project design. A.D.M., E.D.G., M.A.H., C.R.P., conducted the standardization of adapted in house RBD-IgG ELISA. A.K.S.L., J.A.L.L., M.A.H., handle and management biologicals samples. A.K.S.L., J.A.L.L., M.A.H., conducted the clinical evaluation. H.H.M.C., M.A.H., performed IgG-ELISAs. H.H.M.C., E.D.G, M.A.H., C.R.P, designed and performed avidity IgG ELISAs. A.D.M., H.H.M.C., J.P.C.J., V.A.C performed IgG subclasses ELISA. J.P.C.J., calculation and statistical analysis for IgG subclasses-ELISA. H.H.M.C., J.P.C.J., data, and statistical analysis. A.D.M., H.H.M.C., J.P.C.J., and C.R.P. wrote the manuscript. All authors (A.D.M., H.H.M.C., V.A.C., A.K.S.L., J.A.L.L., E.D.G., M.A.H., J.P.C.J., C.R.P.) reviewed and edited the manuscript.

\section{Competing interests}

The authors declare no competing interest within the scope of this manuscript.

\section{References}

1. Zheng, J. SARS-CoV-2: an Emerging Coronavirus that Causes a Global Threat. Int. J. Biol. Sci. (2020) doi:10.7150/ijbs.45053.

2. Zhang, J. jin et al. Clinical characteristics of 140 patients infected with SARS-CoV-2 in Wuhan, China. Allergy Eur. J. Allergy Clin. Immunol. (2020) doi:10.1111/all.14238.

3. Chen, N. et al. Epidemiological and clinical characteristics of 99 cases of 2019 novel coronavirus pneumonia in Wuhan, China: a descriptive study. Lancet (2020) doi:10.1016/S0140-6736(20)302117.

4. Guan, W. et al. Clinical characteristics of 2019 novel coronavirus infection in China. N. Engl. J. Med. (2020) doi:10.1101/2020.02.06.20020974.

5. Menezes, P. de L., Garner, D. M. \& Valenti, V. E. BRAZIL IS PROJECTED TO BE THE NEXT GLOBAL COVID-19 PANDEMIC EPICENTER. medRxiv (2020).

6. Andreoni, M. Coronavirus in Brazil: What You Need to Know. The New York Times https://www.nytimes.com/article/brazil-coronavirus-cases.html (2020).

7. (OMS), W. H. O. COVID-19 Weekly Epidemiological Update. 1;4 (2020). 
8. Guo, L. et al. Profiling Early Humoral Response to Diagnose Novel Coronavirus Disease (COVID-19). Clin. Infect. Dis.71, 778-785 (2020).

9. Astuti, I. Since January 2020 Elsevier has created a COVID-19 resource centre with free information in English and Mandarin on the novel coronavirus COVID-19. The COVID-19 resource centre is hosted on Elsevier Connect, the company 's public news and information . (2020).

10. Tai, W. et al. Characterization of the receptor-binding domain (RBD) of 2019 novel coronavirus: implication for development of RBD protein as a viral attachment inhibitor and vaccine. Cell. Mol. Immunol. (2020) doi:10.1038/s41423-020-0400-4.

11. Walls, A. C. et al. Structure, Function, and Antigenicity of the SARS-CoV-2 Spike Glycoprotein. Cel/181, 281-292.e6 (2020).

12. Jiang, S., Hillyer, C. \& Du, L. Neutralizing Antibodies against SARS-CoV-2 and Other Human Coronaviruses. Trends in Immunology vol. 41 355-359 (2020).

13. Scohy, A. et al. Low performance of rapid antigen detection test as frontline testing for COVID-19 diagnosis. J. Clin. Virol. (2020) doi:10.1016/j.jcv.2020.104455.

14. Cassaniti, I. et al. Performance of VivaDiag COVID-19 IgM/IgG Rapid Test is inadequate for diagnosis of COVID-19 in acute patients referring to emergency room department. Journal of Medical Virology (2020) doi:10.1002/jmv.25800.

15. Castro, R. et al. COVID-19: a meta-analysis of diagnostic test accuracy of commercial assays registered in Brazil. Brazilian J. Infect. Dis.4, 180-187 (2020).

16. Wölfel, R. et al. Virological assessment of hospitalized patients with COVID-2019. Nature(2020) doi:10.1038/s41586-020-2196-x.

17. Wyllie, A. L. et al. Saliva or Nasopharyngeal Swab Specimens for Detection of SARS-CoV-2. N. Engl. J. Med. (2020) doi:10.1056/nejmc2016359.

18. World Health Organization. Laboratory biosafety guidance related to coronavirus disease (COVID19). Interim Guid. (2020).

19. Pieper-Bigelow, C., Strocchi, A. \& Levitt, M. D. Where does serum amylase come from and where does it go? Gastroenterol Clin North Am (1990).

20. Zhao, J. et al. Antibody responses to SARS-CoV-2 in patients of novel coronavirus disease 2019. Clin. Infect. Dis. (2020) doi:10.1093/cid/ciaa344.

21. Wang, P. Combination of serological total antibody and RT-PCR test for detection of SARS-COV-2 infections. J. Virol. Methods (2020) doi:10.1016/j.jviromet.2020.113919.

22. Andersen, K. G., Rambaut, A., Lipkin, W. I., Holmes, E. C. \& Garry, R. F. The proximal origin of SARSCoV-2. Nature Medicine (2020) doi:10.1038/s41591-020-0820-9.

23. Amanat, F. et al. A serological assay to detect SARS-CoV-2 seroconversion in humans. Nat. Med. (2020) doi:10.1038/s41591-020-0913-5.

24. Stadlbauer, D. et al. A detailed protocol for a serological assay to detect SARS-CoV-2 seroconversion in humans: antigen production and test setup. 1-13 (2019). 
25. Huynh, A. et al. Development of a serological assay to identify SARS-CoV-2 antibodies in COVID-19 patients. medRxiv (2020).

26. Nguyen-Contant, P. et al. S protein-reactive IGG and memory B cell production after human SARSCoV-2 infection includes broad reactivity to the S2 subunit. MBio (2020) doi:10.1128/mBio.0199120.

27. H.-I., S., C.-J., W., Y.-F., T. \& C.-Y., C. Fighting COVID-19: A quick review of diagnoses, therapies, and vaccines. Biomed. J. (2020).

28. Padoan, A. et al. IgA-Ab response to spike glycoprotein of SARS-CoV-2 in patients with COVID-19: A longitudinal study. Clin. Chim. Acta (2020) doi:10.1016/j.cca.2020.04.026.

29. Suthar, M. S. et al. Rapid Generation of Neutralizing Antibody Responses in COVID-19 Patients. Cell Reports Med.1, 100040 (2020).

30. Amanat, F. \& Krammer, F. SARS-CoV-2 Vaccines: Status Report. Immunity (2020) doi:10.1016/j.immuni.2020.03.007.

31. Krammer, F. SARS-CoV-2 vaccines in development. Nature (2020) doi:10.1038/s41586-020-2798-3.

32. Zhang, Y. et al. Safety, tolerability, and immunogenicity of an inactivated SARS-CoV-2 vaccine in healthy adults aged 18-59 years: a randomised, double-blind, placebo-controlled, phase $1 / 2$ clinical trial. Lancet Infect. Dis. (2020) doi:10.1016/s1473-3099(20)30843-4.

33. Walsh, E. E. et al. Safety and Immunogenicity of Two RNA-Based Covid-19 Vaccine Candidates. N. Engl. J. Med. (2020) doi:10.1056/nejmoa2027906.

34. Azkur, A. K. et al. Immune response to SARS-CoV-2 and mechanisms of immunopathological changes in COVID-19. Allergy: European Journal of Allergy and Clinical Immunology (2020) doi:10.1111/all.14364.

35. Poland, G. A., Ovsyannikova, I. G. \& Kennedy, R. B. SARS-CoV-2 immunity: review and applications to phase 3 vaccine candidates. Lancet (2020) doi:10.1016/s0140-6736(20)32137-1.

36. Siracusano, G., Pastori, C. \& Lopalco, L. Humoral Immune Responses in COVID-19 Patients: A Window on the State of the Art. Frontiers in Immunology (2020) doi:10.3389/fimmu.2020.01049.

37. Hsueh, P. R., Huang, L. M., Chen, P. J., Kao, C. L. \& Yang, P. C. Chronological evolution of IgM, IgA, IgG and neutralisation antibodies after infection with SARS-associated coronavirus. Clin. Microbiol. Infect. (2004) doi:10.1111/j.1469-0691.2004.01009.x.

38. Ward, S., Lindsley, A., Courter, J. \& Assa'ad, A. Clinical testing for COVID-19. J. Allergy Clin. Immunol.146, 23-34 (2020).

39. Liu, X. et al. Patterns of IgG and IgM antibody response in COVID-19 patients. 9, 1269-1274 (2020).

40. Long, Q. X. et al. Clinical and immunological assessment of asymptomatic SARS-CoV-2 infections. Nat. Med. (2020) doi:10.1038/s41591-020-0965-6.

41. Frasca, D. et al. Effects of age on H1N1-specific serum IgG1 and IgG3 levels evaluated during the 2011-2012 influenza vaccine season. Immun. Ageing (2013) doi:10.1186/1742-4933-10-14. 
42. Benner, S. E. et al. SARS-CoV-2 Antibody Avidity Responses in COVID-19 Patients and Convalescent Plasma Donors. J. Infect. Dis. (2020) doi:10.1093/infdis/jiaa581.

43. Wolf, J. et al. Differences of SARS-CoV-2 serological test performance between hospitalized and outpatient COVID-19 cases. Clin. Chim. Acta (2020) doi:10.1016/j.cca.2020.10.035.

44. Long, Q. X. et al. Antibody responses to SARS-CoV-2 in patients with COVID-19. Nat. Med.26, 845848 (2020).

45. Kweon, O. J. et al. Antibody kinetics and serologic profiles of SARS-CoV-2 infection using two serologic assays. PLoS One (2020) doi:10.1371/journal.pone.0240395.

46. Padoan, A. et al. Analytical performances of a chemiluminescence immunoassay for SARS-CoV-2 IgM/lgG and antibody kinetics. Clinical Chemistry and Laboratory Medicine (2020) doi:10.1515/cclm-2020-0443.

47. Yang, H. S. et al. SARS-CoV-2 antibody characterization in emergency department, hospitalized and convalescent patients by two semi-quantitative immunoassays. Clin. Chim. Acta (2020) doi:10.1016/j.cca.2020.06.004.

48. Rebello, C. J., Kirwan, J. P. \& Greenway, F. L. Obesity, the most common comorbidity in SARS-CoV-2: is leptin the link? Int. J. Obes.44, 1810-1817 (2020).

49. Engin, A. B., Engin, E. D. \& Engin, A. Two important controversial risk factors in SARS-CoV-2 infection: Obesity and smoking. Environmental Toxicology and Pharmacology (2020) doi:10.1016/j.etap.2020.103411.

50. Frasca, D., Diaz, A., Romero, M. \& Blomberg, B. B. Ageing and obesity similarly impair antibody responses. Clinical and Experimental Immunology (2017) doi:10.1111/cei.12824.

51. Frasca, D. et al. Obesity decreases B cell responses in young and elderly individuals. Obesity (2016) doi:10.1002/oby.21383.

52. Frasca, D., Diaz, A., Romero, M. \& Blomberg, B. B. Leptin induces immunosenescence in human B cells. Cell. Immunol. (2020) doi:10.1016/j.cellimm.2019.103994.

53. Michalakis, K. \& Ilias, I. SARS-CoV-2 infection and obesity: Common inflammatory and metabolic aspects. Diabetes Metab. Syndr. Clin. Res. Rev. (2020) doi:10.1016/j.dsx.2020.04.033.

54. Iwasaki, A. \& Yang, Y. The potential danger of suboptimal antibody responses in COVID-19. Nature Reviews Immunology (2020) doi:10.1038/s41577-020-0321-6.

55. Chan, P. K. S. et al. Antibody avidity maturation during severe acute respiratory syndrome-associated coronavirus infection. J. Infect. Dis. (2005) doi:10.1086/430615.

56. Chan, K. H. et al. Use of antibody avidity assays for diagnosis of severe acute respiratory syndrome coronavirus infection. Clin. Vaccine Immunol. (2007) doi:10.1128/CVI.00056-07.

57. Cavacini, L. A., Kuhrt, D., Duval, M., Mayer, K. \& Posner, M. R. Binding and neutralization activity of human IgG1 and IgG3 from serum of HIV-infected individuals. AIDS Res. Hum. Retroviruses (2003) doi:10.1089/088922203769232584. 
58. Mazzini, L. et al. Comparative analyses of SARS-CoV-2 binding $(\operatorname{lgG}, \lg M, \lg A)$ and neutralizing antibodies from human serum samples. bioRxiv (2020).

59. Kawano, Y., Noma, T. \& Yata, J. Regulation of human IgG subclass production by cytokines. IFNgamma and IL- 6 act antagonistically in the induction of human IgG1 but additively in the induction of IgG2. J. Immunol. (1994).

60. Satoguina, J. S., Weyand, E., Larbi, J. \& Hoerauf, A. T Regulatory-1 Cells Induce IgG4 Production by B Cells: Role of IL-10. J. Immunol. (2005) doi:10.4049/jimmunol.174.8.4718.

61. Greiner, M. Two-graph receiver operating characteristic (TG-ROC): Update version supports optimisation of cut-off values that minimise overall misclassification costs. Journal of Immunological Methods (1996) doi:10.1016/0022-1759(96)00013-0.

62. Pullen, G. R., Fitzgerald, M. G. \& Hosking, C. S. Antibody avidity determination by ELISA using thiocyanate elution. J. Immunol. Methods (1986) doi:10.1016/0022-1759(86)90268-1.

63. Nagao-Dias, A. T. et al. Salivary anti-PGL IgM and IgA titers and serum antibody IgG titers and avidities in leprosy patients and their correlation with time of infection and antigen exposure. Brazilian J. Infect. Dis. (2007) doi:10.1590/S1413-86702007000200009.

64. Santana, S. S. et al. Analysis of IgG subclasses (IgG1 and $\operatorname{lgG} 3$ ) to recombinant SAG2A protein from Toxoplasma gondii in sequential serum samples from patients with toxoplasmosis. Immunol. Lett. (2012) doi:10.1016/j.imlet.2012.02.008.

65. Miot, H. A. Avaliação da normalidade dos dados em estudos clínicos e experimentais. Jornal Vascular Brasileiro (2017) doi:10.1590/1677-5449.041117.

\section{Tables}

\section{Table 1}

Characteristics of 47 enrolled patients. 


\begin{tabular}{|c|c|c|c|}
\hline Characteristics & All patients $(\mathrm{N}=47)$ & Recovered $(\mathrm{N}=35)$ & Dead $(N=12)$ \\
\hline Age, Median (range) & $59(33-82)$ & $55(33-77)$ & $64(53-82)$ \\
\hline \multicolumn{4}{|l|}{ Sex } \\
\hline Male & 29/47 (61.7) & $21 / 35(60)$ & $8 / 12(66.67)$ \\
\hline Female & $18 / 47(38.3)$ & $14 / 35(40)$ & $4 / 12(33.33)$ \\
\hline \multicolumn{4}{|l|}{ Clinical Severity } \\
\hline Mild & 10/47 (21.28) & 10/35 (28.57) & 0 \\
\hline Severe & $37 / 47$ (78.72) & 25/35 (71.43) & $12(100)$ \\
\hline \multicolumn{4}{|l|}{ Symptoms } \\
\hline Fever & $39 / 47$ (82.98) & $31 / 35$ (88.57) & $8 / 12(66.67)$ \\
\hline Dry Cough & $39 / 47$ (82.98) & $31 / 35$ (88.57) & $8 / 12(66.67)$ \\
\hline Dyspnea & $34 / 47(72.34)$ & 25/35 (71.43) & $9 / 12(75)$ \\
\hline Myalgia & $20 / 47(42.55)$ & 16/35 (45.71) & $4 / 12(33.33)$ \\
\hline Acute Respiratory Insufficiency & $10 / 47(21.28)$ & $7 / 35(20)$ & $3 / 12(25)$ \\
\hline Headache & 6/47 (12.77) & $6 / 35(17.14)$ & 0 \\
\hline Thromboembolism & $5 / 47(10.64)$ & $4 / 35(11.43)$ & $1 / 12(8.33)$ \\
\hline Diarrhea & 4/47 (8.52) & $3 / 35(8.57)$ & $1 / 12(8.33)$ \\
\hline Pneumonia & $3 / 47(6.39)$ & $3 / 35(8.57)$ & 0 \\
\hline Chill & $2 / 47(4.26)$ & $1 / 35(2.86)$ & $1 / 12(8.33)$ \\
\hline Asthenia & $2 / 47(4.26)$ & $1 / 35(2.86)$ & 1/12 (8.33) \\
\hline Odynophagia & $1 / 47(2.13)$ & $1 / 35(2.86)$ & 0 \\
\hline Nasal Obstruction & $1 / 47(2.13)$ & $1 / 35(2.86)$ & 0 \\
\hline Respiratory Distress & $1 / 47(2.13)$ & $1 / 35(2.86)$ & 0 \\
\hline Dysuria & $1 / 47(2.13)$ & $1 / 35(2.86)$ & 0 \\
\hline Sweating & $1 / 47(2.13)$ & $1 / 35(2.86)$ & 0 \\
\hline Anosmia & $1 / 47(2.13)$ & $1 / 35(2.86)$ & 0 \\
\hline Sore throat & $1 / 47(2.13)$ & $1 / 35(2.86)$ & 0 \\
\hline Malaise & $1 / 47(2.13)$ & 0 & 1/12 (8.33) \\
\hline Hyporexia & $1 / 47(2.13)$ & 0 & 1/12 (8.33) \\
\hline
\end{tabular}




\begin{tabular}{|c|c|c|c|}
\hline \multicolumn{4}{|l|}{ Coexisting Disorders } \\
\hline Obesity & 25/47 (53.19) & 17/35 (48.57) & $8 / 12(66.67)$ \\
\hline Hypertension & 23/47 (48.94) & 17/35 (48.57) & $6 / 12(50)$ \\
\hline Diabetes & 16/47 (30.04) & 11/35 (31.43) & $5 / 12(41.67)$ \\
\hline HIV & $3 / 47$ (6.39) & 2/35 (5.71) & 1/12 (8.33) \\
\hline Deep vein thrombosis & $3 / 47$ (6.39) & $2 / 35(5.71)$ & 1/12 (8.33) \\
\hline COPD & $3 / 47$ (6.39) & $2 / 35(5.71)$ & 1/12 (8.33) \\
\hline Hypothyroidism & 2/47 (4.26) & $1 / 35(2.86)$ & 1/12 (8.33) \\
\hline Prostatic Hypertrophy & 2/47 (4.26) & $1 / 35(2.86)$ & 1/12 (8.33) \\
\hline Stroke & 2/47 (4.26) & $1 / 35(2.86)$ & 1/12 (8.33) \\
\hline Asthma & 2/47 (4.26) & 2/35 (5.71) & 0 \\
\hline Pre-existing Lesions & $1 / 47(2.13)$ & $1 / 35(2.86)$ & 0 \\
\hline Tinea Cruris & $1 / 47(2.13)$ & 1/35 (2.86) & 0 \\
\hline Sleep apnea & $1 / 47(2.13)$ & $1 / 35(2.86)$ & 0 \\
\hline Epilepsy & $1 / 47(2.13)$ & $1 / 35(2.86)$ & 0 \\
\hline Hepatitis B & $1 / 47(2.13)$ & $1 / 35(2.86)$ & 0 \\
\hline Encephalitis & $1 / 47(2.13)$ & $1 / 35(2.86)$ & 0 \\
\hline Cardiac Insufficiency & $1 / 47(2.13)$ & $1 / 35(2.86)$ & 0 \\
\hline Kidney Transplant & $1 / 47(2.13)$ & $1 / 35(2.86)$ & 0 \\
\hline None & 4/47 (8.52) & $4 / 35(11.43)$ & 0 \\
\hline \multicolumn{4}{|l|}{ Treatment } \\
\hline Antibiotics & 45/47 (95.74) & $33 / 35$ (94.29) & $12(100)$ \\
\hline Antiviral & 24/47 (51.06) & $17 / 35$ (48.57) & 7/12 (58.33) \\
\hline Corticoids & 16/47 (30.04) & 12/35 (34.29) & 4/12 (33.33) \\
\hline Anticoagulants & 13/47 (27.67) & 10/35 (28.57) & $3 / 12(25)$ \\
\hline Chloroquine & 9/47 (19.15) & $7 / 35(20)$ & $2 / 12(16.67)$ \\
\hline Immunosuppressant & $1 / 47(2.13)$ & $1 / 35(2.86)$ & 0 \\
\hline Vasodilator & $1 / 47(2.13)$ & $1 / 35(2.86)$ & 0 \\
\hline
\end{tabular}


a
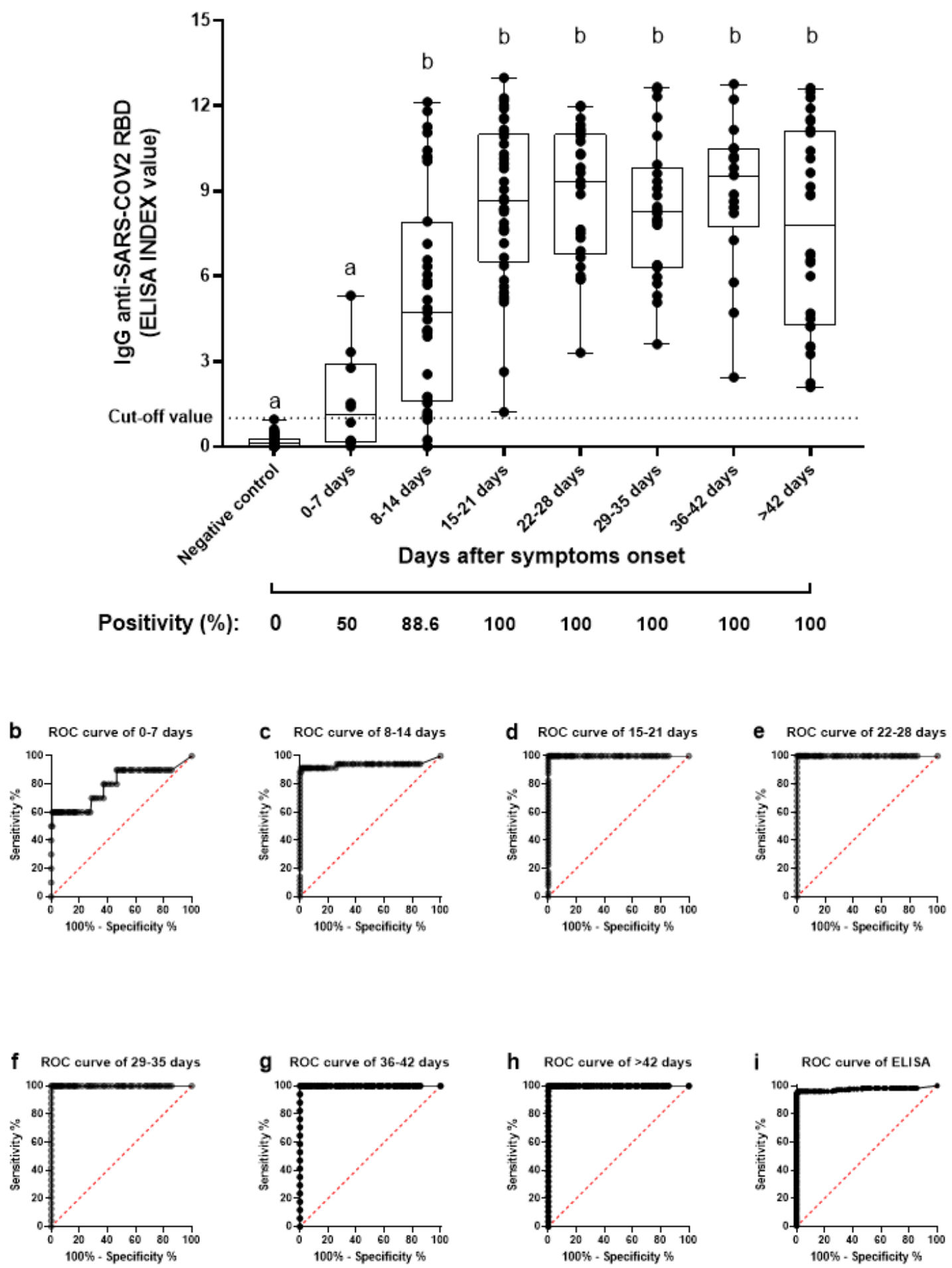

Figure 1

RBD-specific IgG antibody levels in symptomatic patients with SARS-CoV-2. (a). Levels of antibodies against SARS-CoV-2 RBD in patients at different times after symptom onset in 176 samples from 47 patients. Negative samples to COVID-19 (118 samples) were used as a negative control of ELISA. The 
ELISA data were expressed as ELISA Index (EI). Aligned dot plots and boxplots show El values (dots), medians (middle line), third and first quartiles (boxes), while the whiskers display the minimum and maximum values. The dashed line indicates the cut-off value. Different letters $(a, b)$ indicate statistical differences between each time in comparison with negative control determined by Kruskal-Wallis and Dunn's multiple comparisons test $(P<0.05)$. The positivity in each time was expressed as a percentage (\%). (b-i). Receiver operating characteristic (ROC) curves show the RDB IgG-ELISA's diagnostic performance in each time analyzed.

a

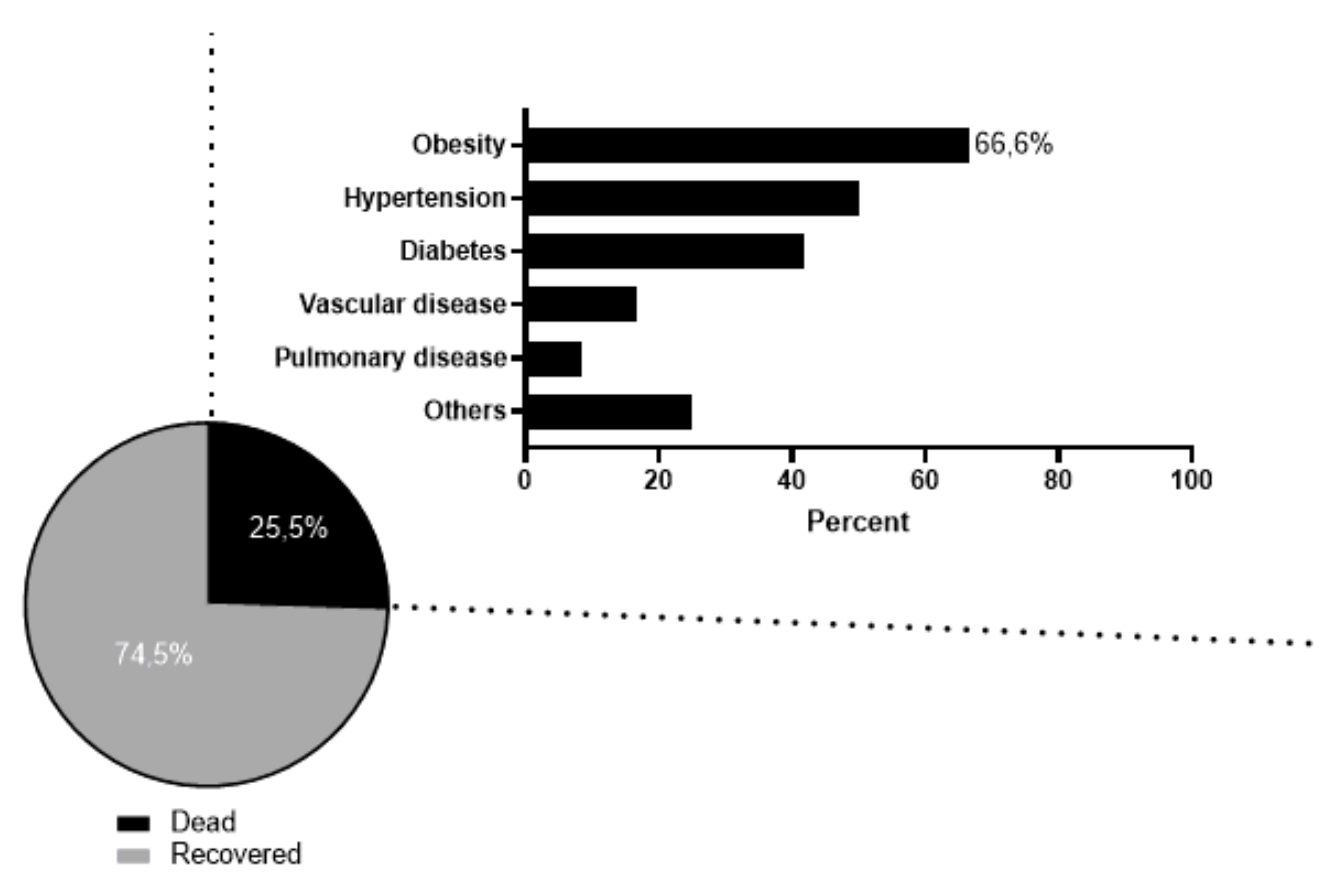

b

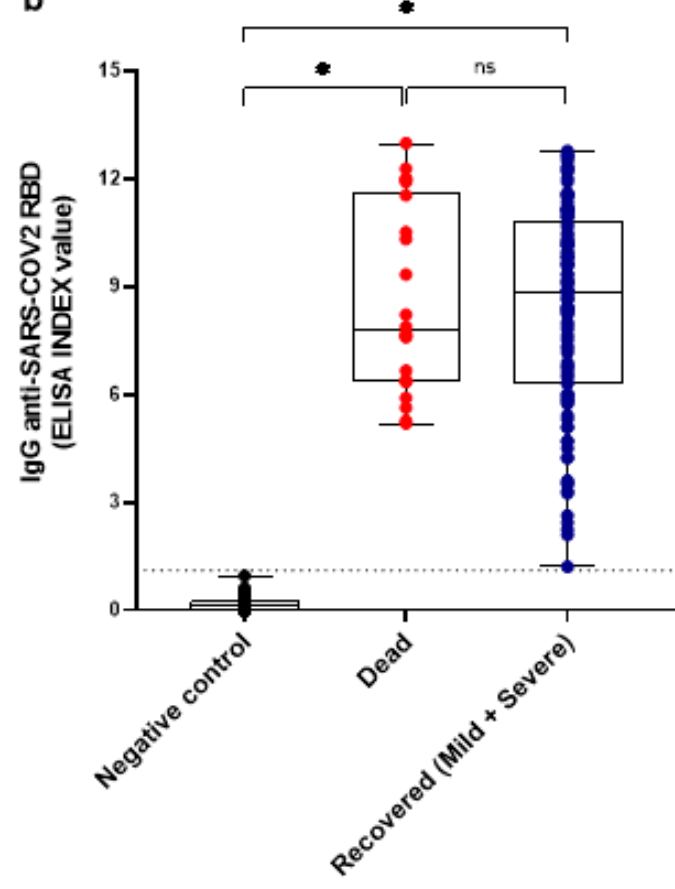

C

Recovered patients

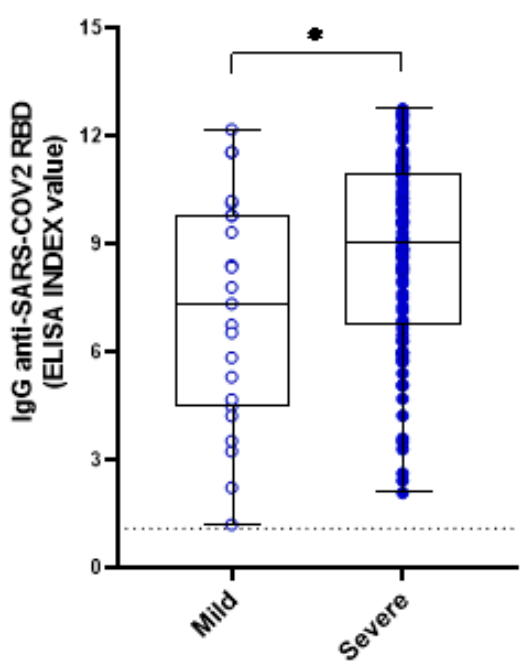




\section{Figure 2}

IgG antibody levels to SARS-CoV-2 RDB in patients who recovered (mild or severe) or who died with COVID-19. (a) Percentage of patients who recovered or died of COVID-19 and main comorbidities diseaseassociated. A detailed description of other comorbidities was shown in table 01. (b) Levels of antibodies to SARS-CoV-2 RDB in patients who recovered (blue) or died (red) of COVID-19 or negative controls (black) were expressed as El. (c) Paired analysis of RDB-specific IgG levels in survived patients displayed mild and severe clinical presentation of COVD-19. Aligned dot plots and boxplots show El values (dots), medians (middle line), third and first quartiles (boxes), while the whiskers display the minimum and maximum values. The dashed lines indicate the cut-off values. Statistically, differences were determined by Kruskal-Wallis and Dunn's multiple comparisons test or Mann-Whitney test when appropriate $\left({ }^{*} \mathrm{P}<\right.$ $0,05)$. 

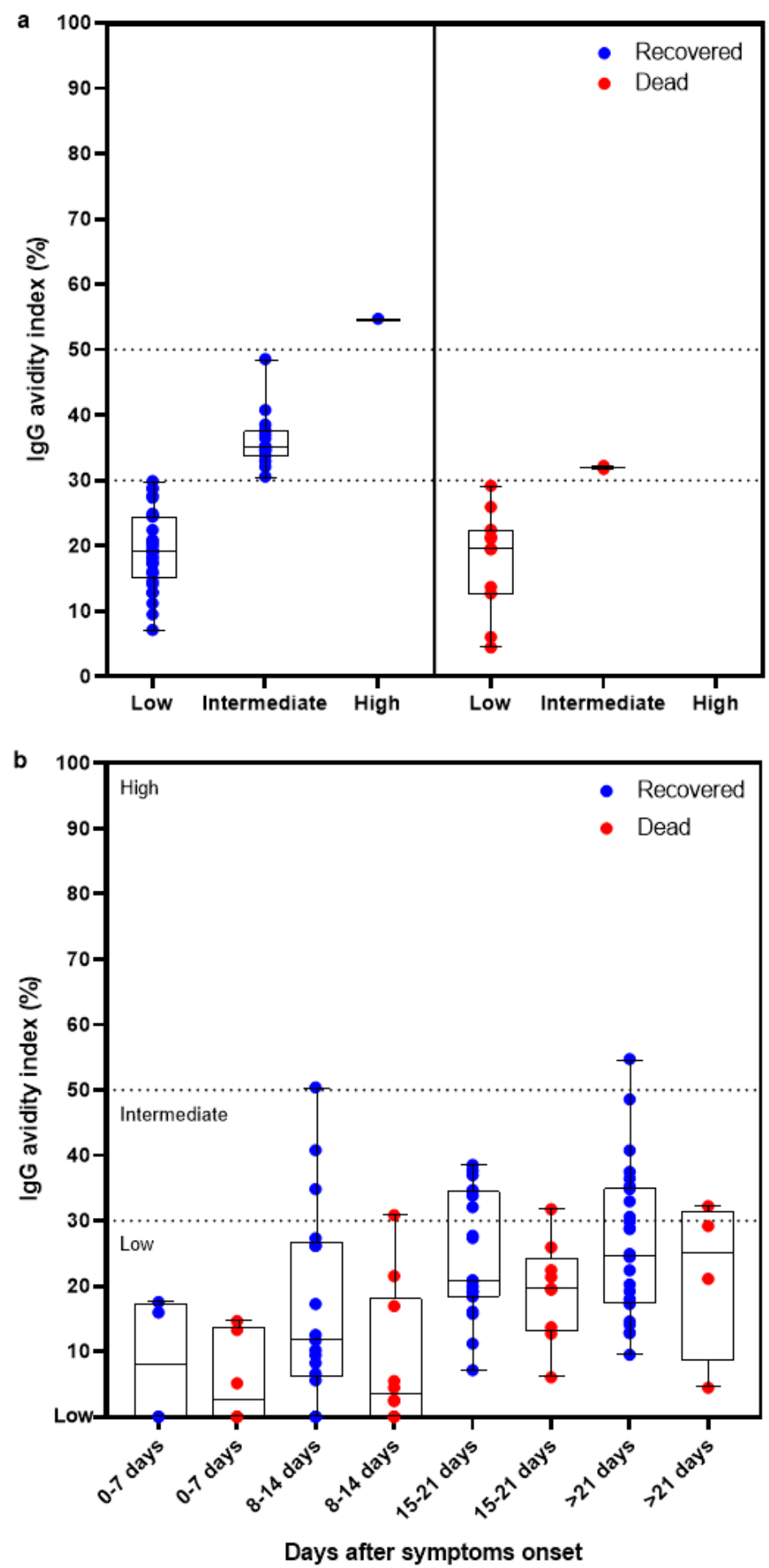

Figure 3

Binding strength of IgG antibody to SARS-CoV-2 RDB (avidity) in COVID-19 patients. (a) IgG antibody avidity in patients who recovered (blue) or who died (red) with COVID-19. The IgG antibody avidity was expressed as the Avidity Index (Al \%). The dash lines indicate the avidity range (low, intermediate, and high). The percentage of serum samples in each avidity range is indicated (\%). (b) IgG antibody avidity to RDB concerning the time after symptom onset in recovered or dead groups. Aligned dot plots and 
boxplots show Al values (dots), medians (middle line), third and first quartiles (boxes), while the whiskers display the minimum and maximum values. Statistically, differences were analyzed by Kruskal-Wallis and Dunn's multiple comparisons test or Mann-Whitney test when appropriate $(P<0,05)$.

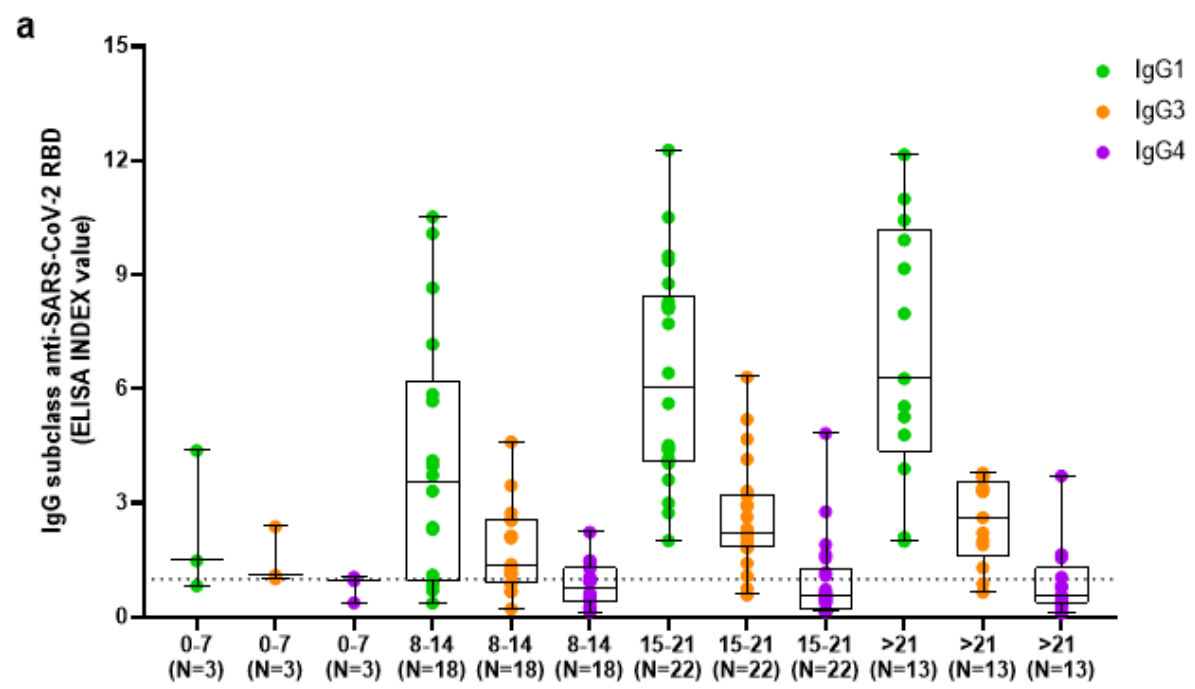

Days after symptoms onset

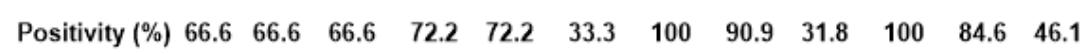
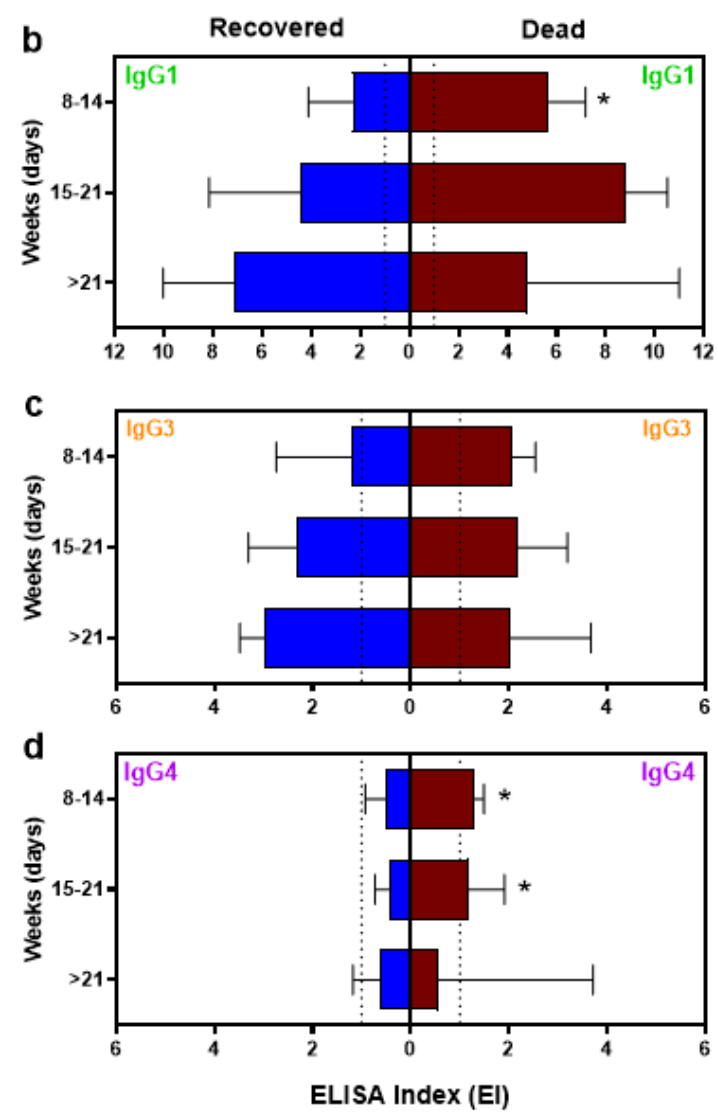

Figure 4

Analyses of IgG subclasses to SARS-CoV-2 RBD in patients with COVID-19. (a) Kinetics of IgG1, IgG3, and IgG4 in COVID-19 patients in different time-points post the onset of the symptoms. The data were 
expressed as El (dots), medians (middle line), third and first quartiles (boxes), while the whiskers display the minimum and maximum values. Numbers of patients $(\mathrm{N})$ are indicated underneath. The positivity, in each time and respective IgG subclasses, was indicated as a percentage (\%). Comparison of IgG1(b), IgG3 (c), and IgG4 (d) in patients who recovered (blue) and who died (red) with COVID-19. Boxplots show the median of El values and interquartile ranges. The dashed lines indicate the cut-off values.

Statistically, differences between groups were determined by the Mann-Whitney test each time $(* \mathrm{P}<$ $0,05)$.

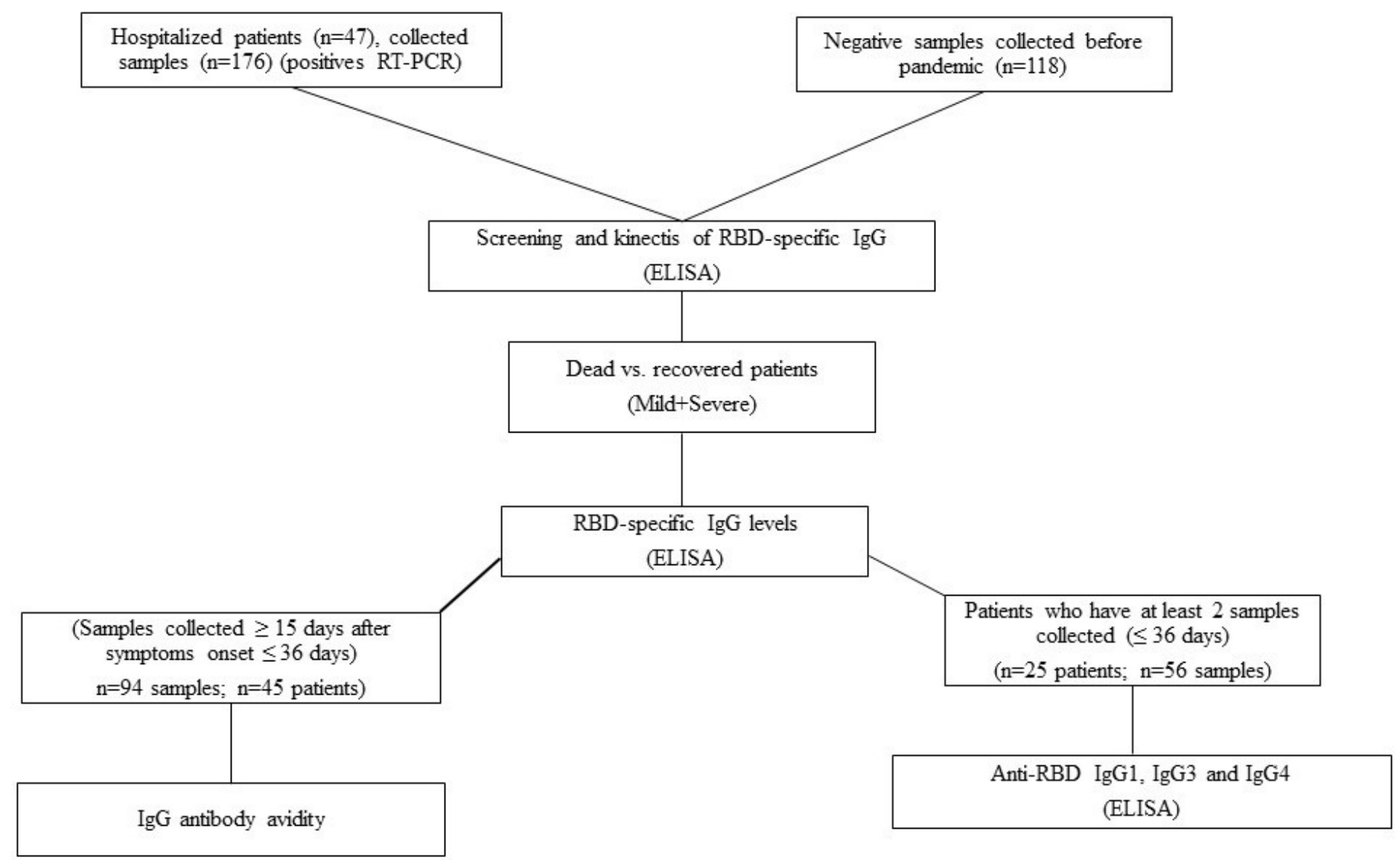

\section{Figure 5}

Flow chart representative of the proposed study design. 